(F) SHUTTLE-BORNE ASTRO MISSIONS 


\title{
ULTRAVIOLET POLARIMETRY
}

\author{
A.D. CODE, K.H. NORDSIECK, C.M. ANDERSON \\ University of Wisconsin
}

\begin{abstract}
Interstellar polarization and polarization of the sky background is predicted to be significantly smaller in the ultraviolet than in the optical region of the spectrum; while intrinsic polarization is expected to be greater. For these reasons alone ultraviolet polarimetry should provide a powerful diagnostic tool. To date such polarization measurements have not been undertaken. The Wisconsin Ultraviolet Photopolarimeter Experiment (WUPPE) will be the first to carry out a program of UV spectropolarimetry. WUPPE is one of the four ASTRO-1 payloads. It consists of a half meter aperture Cassegrain telescope feeding a spectropolarimeter capable of measuring spectral energy distribution and linear and circular polarization in the spectral region from about $3300 \AA$ to $1400 \AA$ with spectral resolutions from about $4 \AA$ to $40 \AA$, depending upon the mode of observation and nature of the source. The effective area of WUPPE is approximately $100 \mathrm{~cm}^{2}$ at $2000 \AA$ and the faint stellar limit for measurement of all four Stokes parameters is about 16 magnitude. The design provides for a large dynamic range and the high precision required for accurate polarimetry. Launch of the first ASTRO Mission is expected in May of 1990 .

The science goals for WUPPE include studies of ultraviolet interstellar polarization, intrinsic polarization of a wide variety of stellar types and measurements of polarization of selected extragalactic objects. A description of WUPPE and simulations of expected data will be presented. The paper will also discuss a wide field imaging survey polarimeter (WISP) now undergoing development for a sounding rocket payload at the University of Wisconsin. (This work is supported by NASA contract NAS5-26777).
\end{abstract}

Y. Kondo (ed.), Observatories in Earth Orbit and Beyond, 291.

(C)1990 Kluwer Academic Publishers. Printed in The Netherlands. 